\title{
Dependence of Magnetic Susceptibility on Dislocation Density in Tensile Deformed Iron and Mn-steel
}

\author{
Koh YAEGASHI \\ Formerly Nondestructive Evaluation and Science Research Center, Faculty of Engineering, Iwate University, Now at Yodogawa \\ Steel Works, Ltd., 9 Showa-cho, Kure 737-0027 Japan.
}

(Received on July 4, 2006; accepted on October 20, 2006)

\begin{abstract}
Dependence of the coefficient $c$ of magnetic susceptibility on dislocation density was investigated in tensile deformed polycrystalline Fe and Mn-steel (JIS SFVQ-1A). The dislocation density was measured by transmission electron microscopy (TEM). The coefficient $c$ was analyzed from hysteresis loops, and found to be proportional to dislocation density. It is concluded that the observed dependence of $c$ is valid when there exist homogeneously distributed dislocations. The observed dependence of $c$ on dislocation density would be useful for nondestructive evaluation of dislocation structure in practical steel.
\end{abstract}

KEY WORDS: dislocation density; magnetic susceptibility.

\section{Introduction}

Magnetic properties in ferromagnetic metals, such as Fe, $\mathrm{Co}$ and $\mathrm{Ni}$, are characterized by magnetic susceptibility, coercive force and so on. Magnetic susceptibility $\chi$ is sensitive to microstructure in materials. Magnetization process proceeds by mainly domain wall motion in low magnetic field, and magnetic moments rotation in high field. Magnetic susceptibility near coercive force is characterized by domain wall motion. ${ }^{1)}$ In high magnetic field near saturation, magnetization process proceeds by magnetic moments rotation, $\chi$ is a parameter of local anisotropy around dislocation, and written by

$$
\chi=\frac{c}{H^{3}}
$$

The coefficient $c$ indicates a state of internal stress because magnetic moments around dislocation are deviated from magnetic field direction through magneto-elastic coupling. The dependence of the coefficient $c$ on dislocation density has been studied by Kronmüller and others. ${ }^{2,3)}$ They have reported that the coefficient $c$ is increased with increasing internal stress amplitude, and proportional to shear stress $\tau^{2}$ in tensile deformed $\mathrm{Ni}, \mathrm{Co}$ and $\mathrm{Ni}-20 \% \mathrm{Co}$ single crystals at work hardening stage II. In general, shear stress is given by $\tau \propto \alpha \mu b \rho^{1 / 2}$ ( $\alpha$ : a constant from 0.1 to $1, \mu$ : the shear modulus, $b$ : the Burgers vector amplitude, $\rho$ : dislocation density). Accordingly, the relation $c \propto \rho$ is derived, and they established theoretically. In addition, the coefficient $c$ is sensitive to a change of dislocation distribution due to fatigue deformation. They have proposed dislocation structure analyzing by measuring the coefficient $c{ }^{4)}$

Recently, Takahashi et al. have reported that magnetic susceptibility in a low magnetic field less than 100 Oe is given by Eq. (1) in tensile deformed Fe single crystal, poly- crystalline Fe and ASTM A533B steel. ${ }^{5)}$ The coefficient $c$ in a low magnetic field is increased due to tensile deformation. It indicates the coefficient $c$ at low field depends on dislocation density similarly as in a high magnetic field. Therefore, it is possible to evaluate dislocation structure in ferromagnetic materials by measuring the coefficient $c$ without high field. However, the relation between the coefficient $c$ and dislocation density has not been discussed sufficiently. It is necessary for establishment of a new evaluation method by measurement of the coefficient $c$ to clarify this relation.

The purpose of the present study is to clarify the relation between the coefficient $c$ and dislocation density by direct observation and modeling calculation of magnetic moment rotation.

\section{Experimental Procedure}

Studied materials were polycrystalline $\mathrm{Fe}$ and $\mathrm{Mn}$-steel (JIS SFVQ-1A). SFVQ-1A steel is usually used in pressure vessel. This steel is similar to A533B steel in chemical composition and heat treatment. The chemical compositions of the materials are shown in Table 1. Cold rolled sheets of polycrystalline Fe was annealed at $993 \mathrm{~K}$ for $1 \mathrm{~h}$. Mn-steel was austenitized at $1133-1163 \mathrm{~K}$ for $2 \mathrm{~h}$, and water quenched. Subsequently, tempering was carried out at 923-938 K for $2 \mathrm{~h}$. These two materials had different grain sizes, which were $20 \mu \mathrm{m}$ for polycrystalline Fe and $10 \mu \mathrm{m}$ for Mn-steel. Tensile test specimens were cut out by electric discharge method from sheets of the materials. The dimension of test specimen was $60 \mathrm{~mm}$ in length with $20 \mathrm{~mm}$ in width and $3 \mathrm{~mm}$ in thickness. Tensile deformation was carried out at room temperature with the strain rate of $0.2 \% / \mathrm{min}$. After tensile deformation, those samples which had doughnut-shape for magnetic measurement and disk- 
Table 1. Chemical compositions of polycrystalline Fe and Mn-steel.

\begin{tabular}{c|cccccccc|c}
\hline \hline Element(wt. \%) & $\mathrm{C}$ & $\mathrm{Si}$ & $\mathrm{Mn}$ & $\mathrm{P}$ & $\mathrm{S}$ & $\mathrm{Ni}$ & $\mathrm{Mo}$ & $\mathrm{Cr}$ & $\mathrm{Fe}$ \\
\hline Mn-steel (SFVQ-1A) & 0.18 & 0.18 & 1.50 & 0.004 & 0.001 & 0.89 & 0.12 & 0.12 & \multirow{2}{*}{ bal. } \\
\cline { 1 - 7 } Polycrystalline Fe & 0.08 & 0.002 & 0.002 & 0.002 & 0.001 & - & - & - & \\
\hline \hline
\end{tabular}

polycrystalline $\mathrm{Fe}$

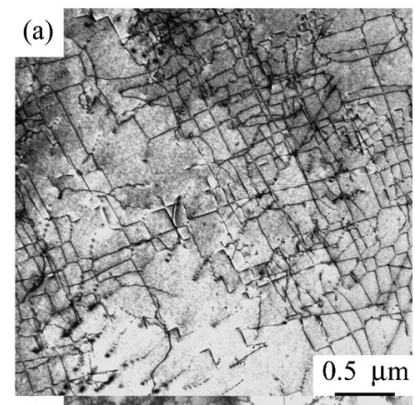

(b)
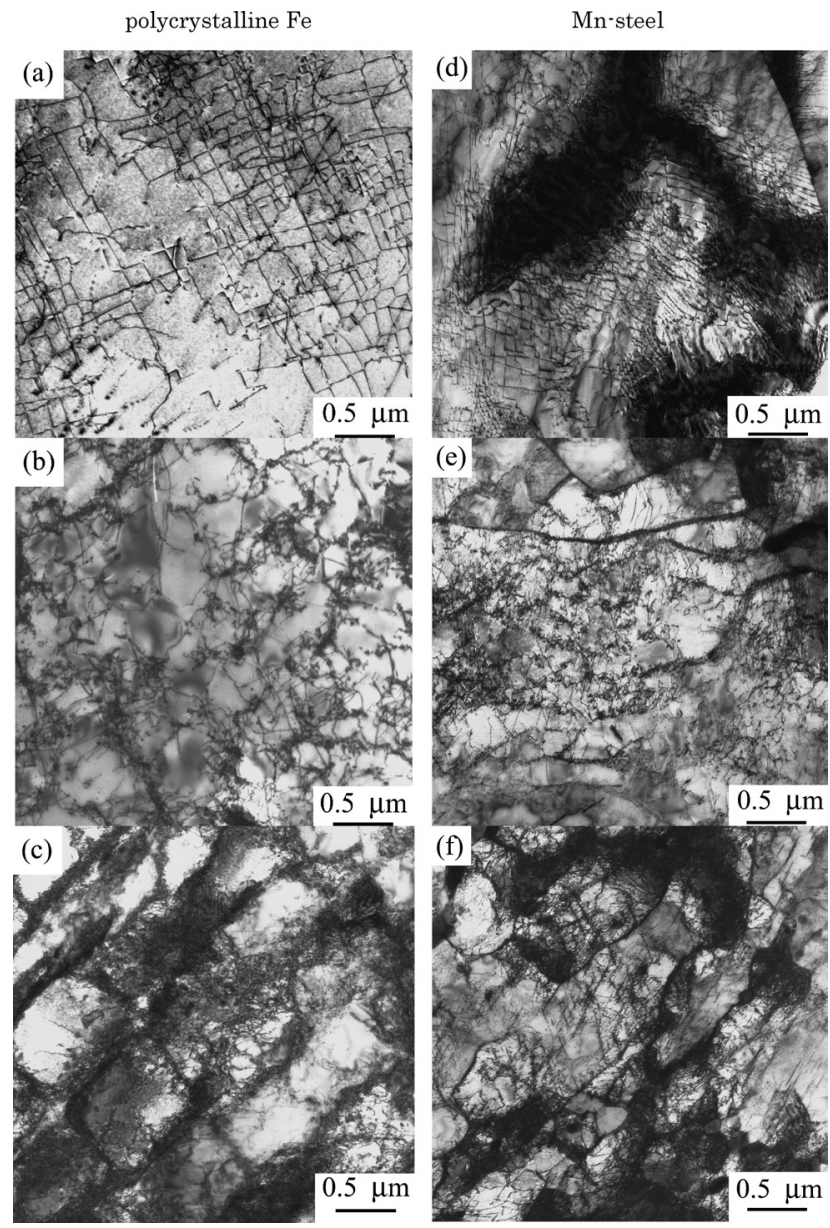

Fig. 1. TEM micrographs of polycrystalline Fe (a) as annealed, (b) deformed unto $290 \mathrm{MPa}$, (c) $370 \mathrm{MPa}$, and Mn-steel (d) as received, (e) $480 \mathrm{MPa}$, (f) $540 \mathrm{MPa}$ true stress.

shape for dislocation density observation were cut out. The doughnut-shape sample was of $10 \mathrm{~mm}$ outside diameter and $5 \mathrm{~mm}$ inside diameter. The excitation coil and the search were wounded 45 and 60 turns, respectively. The diskshape sample was polished mechanically down to $40 \mu \mathrm{m}$, and electro polished with $10 \%$ perchloric acid and $90 \%$ acetic acid mixed electrolyte. Dislocation structure was observed by TEM at $300 \mathrm{kV}$ accelerating voltage.

\section{Results and Discussions}

\subsection{Dislocation Density in Polycrystalline $\mathrm{Fe}$ and Mn-steel}

Tensile samples were deformed differently unto several stresses between the yield stress to tensile strength. The yield stress and tensile strength are 270 and $330 \mathrm{MPa}$ for polycrystalline Fe, 450 and $580 \mathrm{MPa}$ for Mn-steel. Different dislocation structures which depend on the stress level of tensile deformation were observed as shown in Fig. 1. In as annealed polycrystalline $\mathrm{Fe}$, dislocations are distributing homogeneously in a wide area (Fig. 1(a)), while deformed

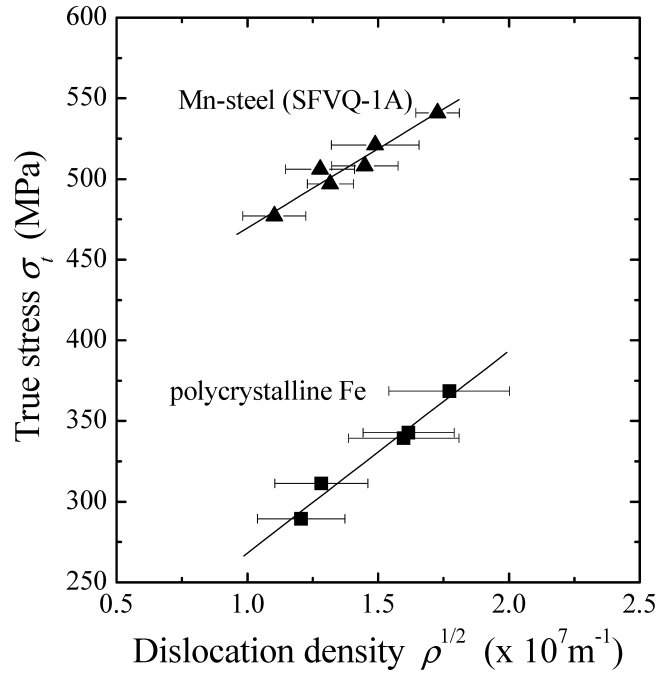

Fig. 2. Dislocation density in tensile deformed polycrystalline $\mathrm{Fe}$ and Mn-steel.

samples showed dislocation tangles and cellular structure (Figs. 1(b)-1(c)). The average diameter of cell is $1 \pm 0.1 \mu \mathrm{m}$. In Mn-steel, spheroidal carbides exist sparsely (Fig. 1(d)). The change of dislocation structure due to tensile deformation is similar as polycrystalline Fe, excepting the cell diameter, which is smaller than $0.5 \mu \mathrm{m}$ (Figs. 1(e)-1(f)). The dislocation density in the cell interior was measured for 20 pictures taken from one TEM sample using Keh method. ${ }^{6)}$ This method gives the dislocation density $\rho$ from the number of intersections of dislocation lines and grid lines, according to the equation of

$$
\rho=\frac{\left\langle n_{\mathrm{h}} / l_{\mathrm{h}}+n_{\mathrm{v}} / l_{\mathrm{v}}\right\rangle_{\mathrm{ave}}}{t}
$$

where $n$ is the number of intersections, $l$ is the length of mesh and $t$ is the thickness of sample. The grid lines are composed of ten horizontal and vertical lines. The sample thickness of $200 \mathrm{~nm}$ was calculated from equi-thick fringes.

Dependence of measured dislocation density on tensile stress for polycrystalline $\mathrm{Fe}$ and $\mathrm{Mn}$-steel are shown in Fig. 2. Measured dislocation density is given with error bars associated with variation of measured value over the observed area. The initial dislocation density is respectively $0.61 \times 10^{14} \mathrm{~m}^{-2}$ for annealed polycrystalline $\mathrm{Fe}$, and $0.68 \times$ $10^{14} \mathrm{~m}^{-2}$ for as-received Mn-steel. In both materials, dislocation densities are increased due to tensile deformation. The relation between dislocation density and true stress is plotted in according to the following Bailey-Hirsch equation

$$
\sigma \propto \alpha \mu b \rho^{1 / 2}
$$

where $\alpha$ is a constant between 0.1 and $1, \mu$ is the shear modulus, $b$ is the magnitude of Burgers vector. The ob- 
served value of the constant $\alpha$ for Mn-steel is nearly equal to that for polycrystalline Fe. For deformed samples applied a high tensile stress, the measured value of dislocation density more than $6 \times 10^{14} \mathrm{~m}^{-2}$ may not be reliable because it is difficult to count the density of tangled dislocations individually. Therefore, the relation between the coefficient $c$ and dislocation density is discussed for the range of low dislocation density samples lower than $6 \times 10^{14} \mathrm{~m}^{-2}$.

\subsection{The Coefficient $c$ for Deformed Samples}

Figure 3 shows differential magnetic susceptibility $\chi$ obtained from $B-H$ curves of tensile deformed samples. Coercive force is $1.5 \mathrm{Oe}(\log H=0.2)$ for non-deformed polycrystalline $\mathrm{Fe}$, and $6.1 \mathrm{Oe}(\log H=0.8)$ for Mn-steel. The values of magnetic susceptibility $\chi$ show the peak near coercive force in all samples. The peak value becomes low due to deformation. Dislocations act the obstructing sites against domain walls displacement, therefore the magnetic susceptibility is decreased. In addition, the peak value for Mn-steel is lower than for polycrystalline Fe because precipitations and grain boundaries act the obstructing site similar to dislocations.

The coefficient of magnetic susceptibility $c$ is calculated from measured magnetic susceptibility $\chi$ according to Eq. (1) within a limited magnetic field region indicated by arrows. Equation (1) is not valid near 100 Oe because the value of $\chi$ is scattered. The reason for this will be discussed later. In the indicated region, the susceptibility of deformed samples is higher than that of non-deformed sample. The observed coefficient $c$ for polycrystalline Fe and Mn-steel are plotted as a function of dislocation density $\rho$ in Fig. 4. The initial values of the coefficient $c_{\text {ini }}$ for non-deformed polycrystalline $\mathrm{Fe}$ and $\mathrm{Mn}$-steel are $0.1 \times 10^{6}$ and $1.6 \times 10^{6}$ Gauss $\mathrm{Oe}^{2}$, respectively. The relation between the coefficient $c$ and dislocation density $\rho$ is given according to

$$
c=c_{0}+\gamma_{\mathrm{c}} \rho
$$

This relation is also satisfied in a very high field above 1000 Oe. $^{3)}$

\section{Modeling Calculation}

The relation between the measured coefficient $c$ at low field and dislocation density is discussed in the preceding chapter. It is important to discuss the influence of microstructure on $c_{0}$ and $\gamma_{\mathrm{c}}$ for clarifying the relation the coefficient $c$ and dislocation density. However, the measurement error of dislocation density is inevitable and it has influence on reliability of measurement value of $c_{0}$ and $\gamma_{\mathrm{c}}$. In this chapter, the relation is discussed by modeling calculation of magnetic moments orientation to assure the reliability of experimental result.

\subsection{Energies Associated with Magnetic Moment}

Magnetic moments are usually oriented in the easy magnetization direction. When dislocations exist in a ferromagnetic material, magnetic moments are deviated through magneto-elastic coupling. Deviated magnetic moments are oriented in the direction to minimize the magnetic energy that is given by

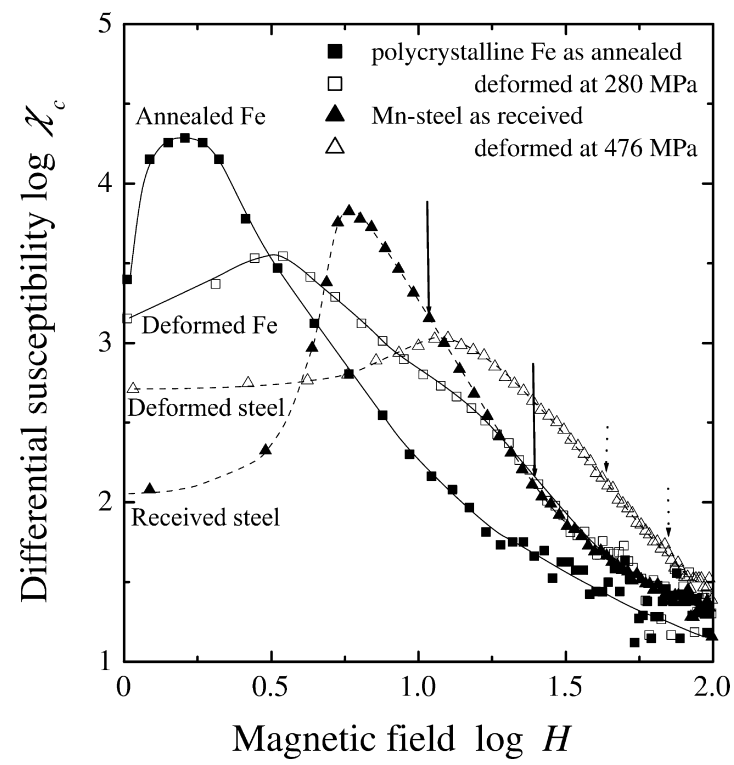

Fig. 3. Differential susceptibility of polycrystalline Fe and Mnsteel.

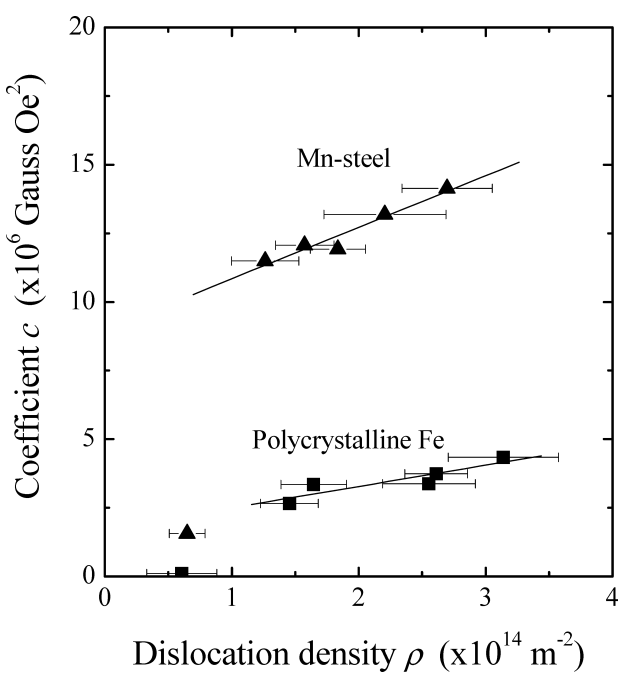

Fig. 4. Relationship between the coefficient $c$ and dislocation density in tensile deformed polycrystalline Fe and Mnsteel.

$$
E_{\mathrm{tot}}=E_{\mathrm{a}}+E_{H}+E_{\sigma}
$$

$E_{\mathrm{a}}$ is the magnetocrystalline anisotropy energy, it becomes minimum when magnetic moment orients in the easy magnetic direction. $E_{H}$ is induced by the applying external field, it is decreased with decreasing the angular difference between the magnetic moment and the magnetic field direction. $E_{\sigma}$ is the magneto-elastic coupling energy, it is influenced from the internal stress field in material. These three terms on the right hand of the equation depend on lattice structure of material. In this study, the assumptions made for calculation are as follows; i) single crystal Fe ii) single magnetic domain iii) the initial direction of magnetic moment is $\langle 001\rangle$, external field direction is $\langle 010\rangle$ parallel to the Burgers vector of dislocation, so that magnetic moments are rotated in (100) plane.

The magnetocrystalline anisotropy energy $E_{\mathrm{a}}$ in (100) plane is given by ${ }^{7)}$ 


$$
E_{\mathrm{a}}=\frac{K_{1}}{8}(1-\cos 4 \theta)+\frac{K_{3}}{128}(3-4 \cos 4 \theta+\cos 8 \theta)
$$

where $K_{1}$ and $K_{3}$ are anisotropy constants, $\theta$ is an angle between magnetic moment and easy direction. $E_{H}$ is induced by applying external field, and given by ${ }^{7)}$

$$
E_{H}=-M_{\mathrm{S}} H \cdot \sin \varphi
$$

where $M_{\mathrm{S}}$ is the saturation magnetization, $\varphi$ is an angle between magnetic moment and external field direction. $E_{\mathrm{a}}$ and $E_{H}$ associated with all magnetic moments are determined by the crystal orientation. Magneto-elastic coupling energy $E_{\sigma}$ is given by ${ }^{8)}$

$$
E_{\sigma}=-\frac{3}{2} \lambda_{100} \sum_{i=1}^{3} \sigma_{i i} \gamma_{i}^{2}-\frac{3}{2} \lambda_{111} \sum_{i \neq j} \sigma_{i j} \gamma_{i} \gamma_{j}
$$

where $\lambda_{100}$ and $\lambda_{111}$ are magnetostriction constants, $\sigma_{i i}$ and $\sigma_{i j}$ are internal stress tensors, $\gamma_{i}$ and $\gamma_{j}$ are directional cosines of magnetization. $E_{\sigma}$ depends on the distance between magnetic moment and dislocation because internal stress tensors are a function of position. Although the exchange interaction influences on magnetic orientation, we neglect this for simplification of this calculation.

Internal stress tensors are calculated under the following conditions; i) operative slip systems are $(110)\langle\overline{1} 11\rangle$ and $(110)\langle 1 \overline{1} 1\rangle$. ii) Burgers vector directions of all dislocations are same. iii) the average distance between neighboring dislocations is given by $d=\rho^{-1 / 2}$. Dislocation distribution is illustrated in Fig. 5 with $\langle 100\rangle$ direction on the $x$-axis, $\langle 010\rangle$ direction on the $y$-axis and $\langle 001\rangle$ direction on the $z$-axis. Stress tensors around single dislocation are given by the following equations. ${ }^{9)}$

$$
\begin{aligned}
& \sigma_{y y}=-\frac{\mu b}{2 \pi(1-v)} \frac{Z\left(3 Y^{2}+Z^{2}\right)}{\left(Y^{2}+Z^{2}\right)^{2}} \\
& \sigma_{z z}=\frac{\mu b}{2 \pi(1-v)} \frac{Z\left(Y^{2}-Z^{2}\right)}{\left(Y^{2}+Z^{2}\right)^{2}}
\end{aligned}
$$

(a)

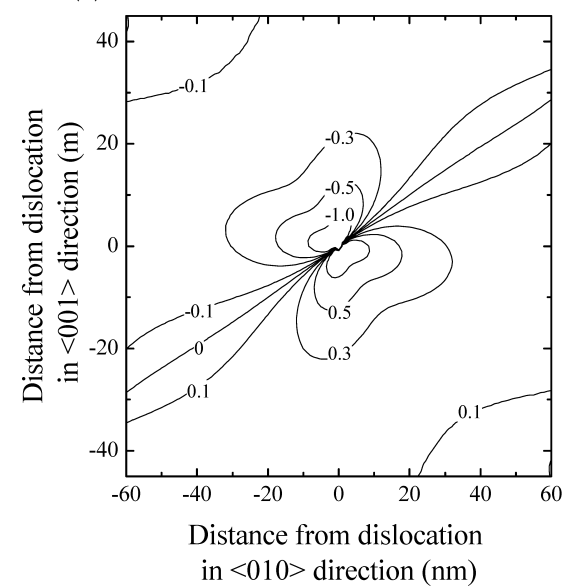

$$
\begin{aligned}
& \sigma_{x x}=v\left(\sigma_{y y}+\sigma_{z z}\right) \\
& \sigma_{y z}=\frac{\mu b}{2 \pi(1-v)} \frac{Z\left(Y^{2}-Z^{2}\right)}{\left(Y^{2}+Z^{2}\right)^{2}} \\
& \sigma_{z x}=\sigma_{x y}=0 \\
& X=\frac{x}{\sqrt{2}}-\frac{y}{\sqrt{2}}, \quad Y=\frac{x}{\sqrt{3}}+\frac{y}{\sqrt{3}}+\frac{z}{\sqrt{3}} \\
& Z=-\frac{x}{\sqrt{6}}-\frac{y}{\sqrt{6}}+\sqrt{\frac{2}{3}} z
\end{aligned}
$$

$v$ is Poisson's ratio. Total stress tensors in crystal are calculated by adding stress tensors of dislocations. Figure 6 shows stress tensor $\sigma_{z z}$ for different dislocation configurations with dislocation density of $1.5 \times 10^{14} \mathrm{~m}^{-2}$ (Fig. 6(a)) and $6.0 \times 10^{14} \mathrm{~m}^{-2}$ (Fig. 6(b)). Stress field of neighbor dislocations overlap each other.

\subsection{Calculation of the Coefficient $c$}

The directions of magnetic moment can locally change due to an increase in dislocation density as shown in Fig. 7. Most magnetic moments are parallel to the easy direction $\langle 001\rangle$. Then application of a magnetic field of $10 \mathrm{Oe}$, these moments are turned to external field direction $\langle 010\rangle$. Several magnetic moments around dislocation are slanted by $1-10$ degree or inverted from $\langle 001\rangle$ to nearly $\langle 00 \overline{1}\rangle$ direc-

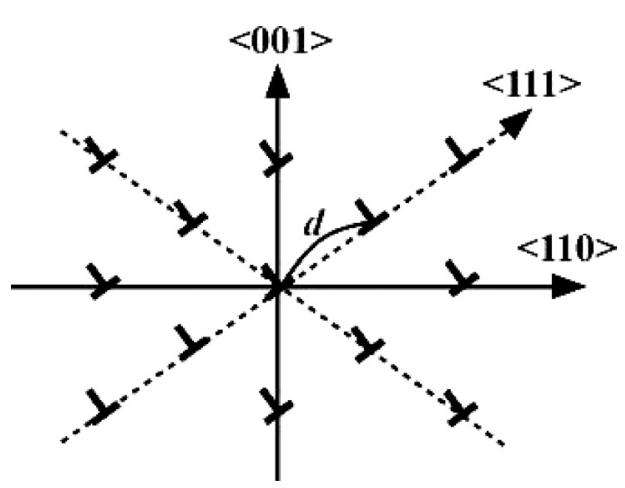

Fig. 5. Schematic illustration of homogeneously distributed dislocations.

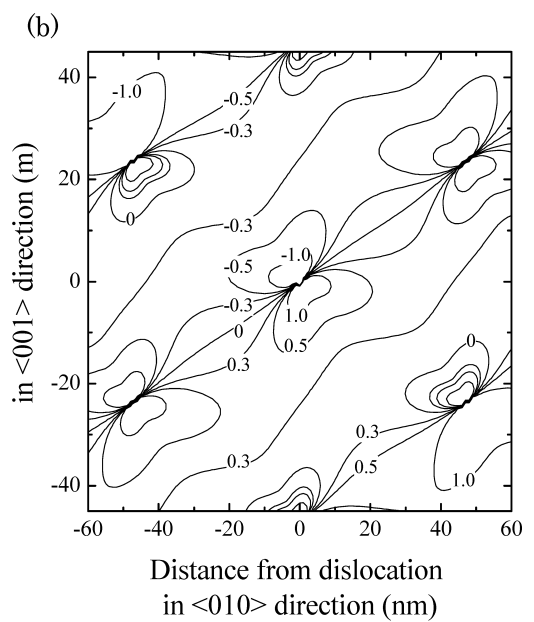

Fig. 6. Stress tensor $\sigma_{z z}\left(\times 10^{9} \mathrm{~Pa}\right)$ for different configurations with different dislocation densities of (a) of $1.5 \times 10^{14} \mathrm{~m}^{-2}$ and (b) and $6.0 \times 10^{14} \mathrm{~m}^{-2}$. Dislocations are distributed homogeneously with the average distance given by $d=\rho^{-1 / 2}$. 
(a)

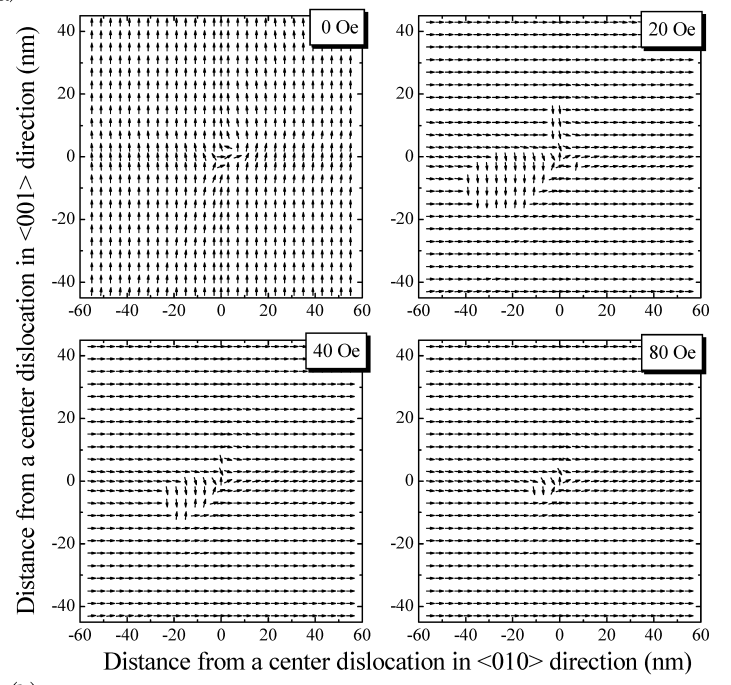

(b)

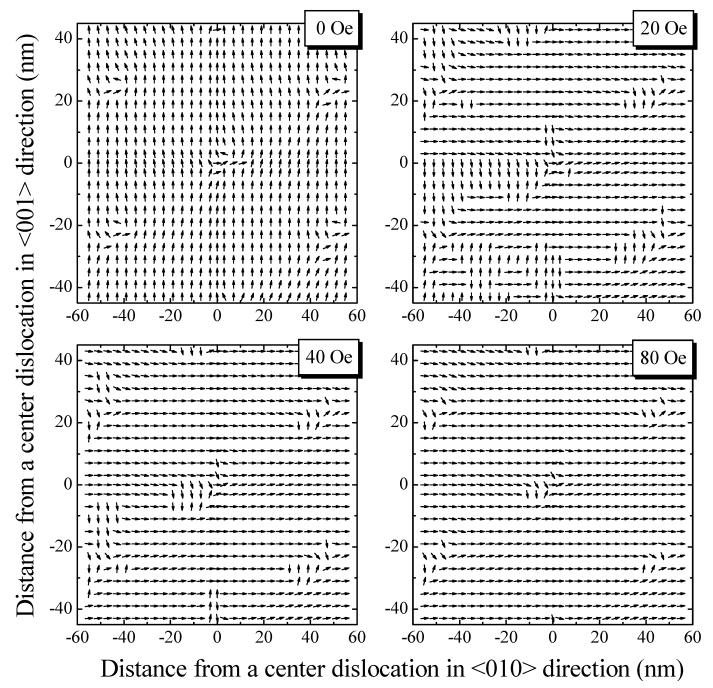

Fig. 7. Magnetic moment arrangement around edge dislocation in calculation models with dislocation density of (a) $1.5 \times 10^{14} \mathrm{~m}^{-2}$ and (b) $6.0 \times 10^{14} \mathrm{~m}^{-2}$

tion. The slanted area of magnetic moments is multiplied with increasing dislocation density. Differential susceptibility is calculated from the rotation angle of magnetic moments as a function of dislocation density under magnetic field between 0 to $5000 \mathrm{Oe}$. The result is plotted as a function of magnetic field in Fig. 8. Equation (1) is valid at two magnetic field regions, low field below 100 Oe and high field above $1000 \mathrm{Oe}$ in Fig. 8. Comparison of the coefficient $c$ between for low magnetic state and high magnetic field state could bring an inaccurate result, because the saturation magnetization is changed by applying very high magnetic field. At very high field, the saturation magnetization is increased with increasing the product of the magnetic field $H$ and the high field susceptibility $\chi_{0}{ }^{7)}$ and the influence of this increase is unable to ignore. Therefore, the relation between the coefficient $c$ and dislocation density $\rho$ for the models is discussed at low magnetic. The coefficient $c$ calculated for low field below 100 Oe is shown in Fig. 9, together with the experimental result. The coefficient $c$ is linearly increased with increasing dislocation density, in other words, increasing the number of deviated magnetic moment. The calculated relation between the coefficient $c$

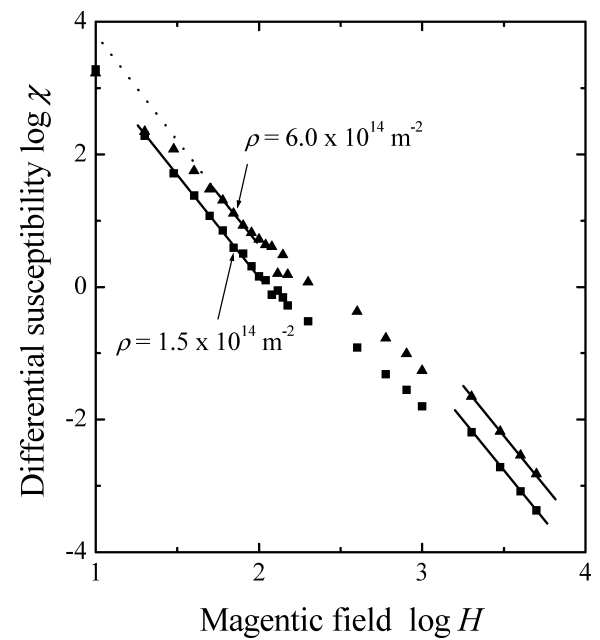

Fig. 8. Calculated differential susceptibility with dislocation density of $1.5 \times 10^{14} \mathrm{~m}^{-2}$ and $6.0 \times 10^{14} \mathrm{~m}^{-2}$.

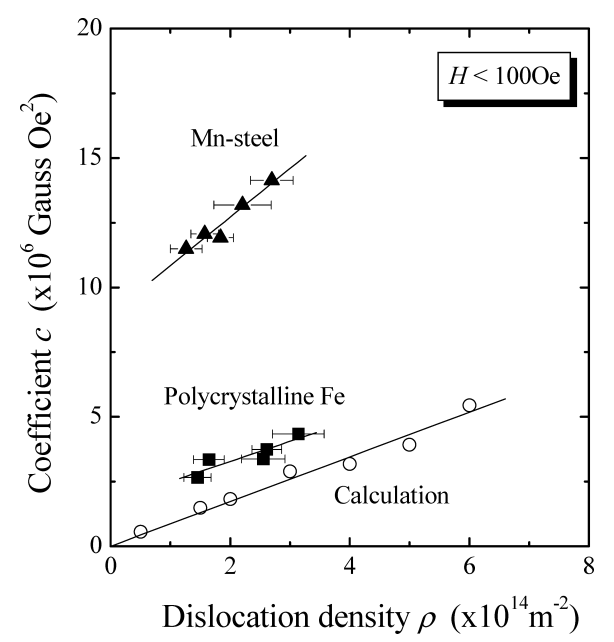

Fig. 9. The calculated coefficient $c$ as a function of dislocation density $\rho$ for low magnetic fields below 100 Oe.

and dislocation density $\rho$ is in reasonable agreement with the experimental result.

At the middle range of magnetic field between 100 and 200 Oe, the value of $\chi$ is scattered same as the experimental result. Magnetic moment orientation around dislocation at the middle magnetic field range is represented in Fig. 10. Most magnetic moments are parallel to magnetic field direction. On the other hand, several magnetic moments around dislocations are deviated from magnetic field direction with large angle. Encircled magnetic moments in Fig. 10 are rotated by $20-80$ degree upon increasing magnetic field. The numbers of magnetic moments rotated unto larger angle under 130 and $150 \mathrm{Oe}$ are smaller than those under other magnetic field levels. Thus susceptibility repeats fluctuation in this magnetic field region.

The values of $c_{0}, \gamma_{\mathrm{c}}$ and $c_{\text {ini }}$ for each sample are given in Table 2. The values of $c_{0}$ and $c_{\text {ini }}$ for Mn-steel are larger than those for polycrystalline Fe and the calculated model. The coefficient $c$ could be affected by lattice defects other than dislocations, for example a grain boundary and a precipitation. Comparison of microstructure structure before deformation between polycrystalline Fe and SFVQ-1A steel, dislocation densities in both materials are same. 
Table 2. Comparison of experimental values of $c_{\text {ini }}, c_{0}$ and $\gamma_{\mathrm{c}}$ for polycrystalline Fe, Mn-steel with calculated ones.

\begin{tabular}{c|ccc}
\hline \hline Parameter & $c_{i n i}$ & $c_{0}$ & $\gamma_{c}$ \\
& $\left(\times 10^{6} \mathrm{Gauss} \mathrm{Oe}^{2}\right)$ & $\left(\times 10^{6} \mathrm{Gauss} \mathrm{Oe}^{2}\right)$ & $\left(\times 10^{-9} \mathrm{Gauss} \mathrm{Oe}^{2} \mathrm{~m}^{-2}\right)$ \\
\hline Polycrystalline Fe & 0.1 & 1.7 & 7.8 \\
\hline Mn-steel & 1.6 & 9.0 & 18.8 \\
\hline Calculated model & - & 0 & 9.2 \\
\hline \hline
\end{tabular}

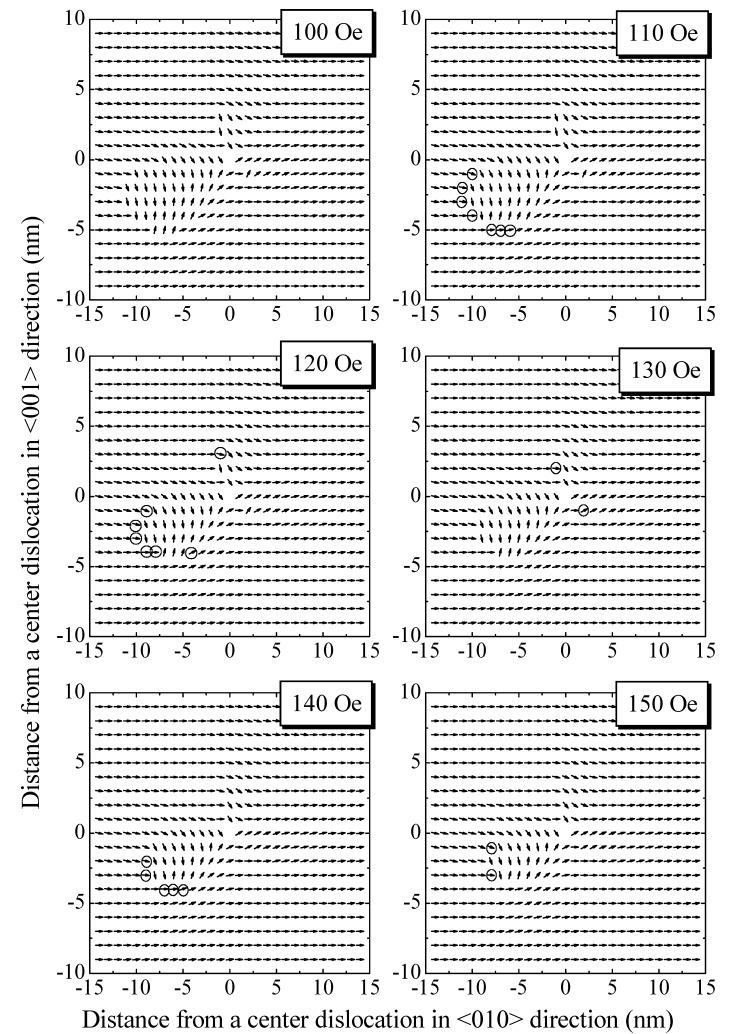

Fig. 10. Magnetic moment arrangement around edge dislocation at several external field in calculation model with dislocation density $1.5 \times 10^{14} \mathrm{~m}^{-2}$.

SFVQ-1A steel has small grain size and spheroidal carbides.

The slope $\gamma_{c}$ for Mn-steel is two times as large as for polycrystalline $\mathrm{Fe}$ and the calculated model. It should make large by growth of cell-structure. Mn-steel has fine cellstructure in comparison with polycrystalline Fe (see Sec. 3.1). In other words, Mn-steel has higher density of cell boundary. Cell boundary would affect on the coefficient $c$ because it has short-range stress field that decays exponentially with the distance from cell boundary. ${ }^{10)}$ The increasing rate of cell boundary density in Mn-steel is larger than polycrystalline Fe, thus $\gamma_{\mathrm{c}}$ should be large. The discrepancy of $c_{\text {ini }}$ and $c_{0}$ between polycrystalline $\mathrm{Fe}$ and $\mathrm{Mn}$-steel would be clarified on the basis of the influence of cell boundary on magnetic susceptibility. When there is no dislocation, $c_{0}$ becomes zero for the calculated result, but the values of $c_{0}$ are not zero in real materials because there exist a lot of lattice defects other than homogeneously distributed dislocations, and also ideally homogeneous distribution of dislocations is rather difficult to occur.

\section{Conclusions}

The coefficient $c$ is calculated for tensile deformed polycrystalline Fe, Mn-steel. From the direct observation results, it is clarified that the coefficient $c$ is proportional to the dislocation density $\rho$ in both materials at low magnetic field under $100 \mathrm{Oe}$. The coefficient $c$ would be affected from a grain boundary, a precipitation and a cell boundary of cell-structure. The effects from these defects on the coefficient $c$ are not discussed sufficiently. It is necessary to comprehend the influence of these defects. In the calculation models that have homogeneously distributed dislocations, the relation between the coefficient $c$ and dislocation density $\rho$ is in reasonable agreement with the experimental result.

\section{REFERENCES}

1) S. Yamaura, Y. Furuya and T. Watanabe: Acta Mater., 49 (2001), 3019.

2) L. J. Dykstra and H. Trauble: Magnetism and Metallurgy, ed. by A. E. Berkowitz and E. Kneller, Academic Press, New York, (1969), 621.

3) H. Kronmüller: Int. J. Nondestruct. Test., 3 (1972), 315.

4) H. Mughrabi, R. Kütterer, K. Lubitz and H. Kronmüller: Phys. Status Solidi (a), 38 (1976), 261.

5) S. Takahashi, J. Echigoya and Z. Motoki: J. Appl. Phys., 87 (2000), 805.

6) A. S. Keh: Direct Observations of Imperfections in Crystals, Interscience, New York, (1961), 213.

7) S. Chikazumi: Physics of Ferromagnetism, 2nd ed., Oxford, (1997).

8) H. Kronmüller and M. Fähnle: Micromagnetism and the Microstructure of Ferromagnetic Solids, Cambridge Univ. Press, Cambridge, (2003), 13.

9) J. P. Hirth and J. Lothe: Theory of Dislocations, 2nd ed., John Wiley \& Sons, New York, (1982), 59.

10) A. P. Sutton and R. W. Balluffi: Interfaces in Crystalline Materials, Clarendon, Oxford, (1995), 115. 